\title{
Investigation of the Scanning Microarc Oxidation Process
}

\author{
Lingqin Xia, ${ }^{1}$ Jianmin Han, ${ }^{1}$ Joseph P. Domblesky, ${ }^{2}$ Zhiyong Yang, ${ }^{1}$ and Weijing Li ${ }^{1}$ \\ ${ }^{1}$ School of Mechanical, Electronic and Control Engineering, Beijing Jiaotong University, Beijing 100044, China \\ ${ }^{2}$ Mechanical Engineering Department, Marquette University, 1515 West Wisconsin Avenue, Milwaukee, WI 53201 1881, USA
}

Correspondence should be addressed to Jianmin Han; jmhan@bjtu.edu.cn

Received 24 January 2017; Revised 21 May 2017; Accepted 28 May 2017; Published 20 June 2017

Academic Editor: Ming-Xing Zhang

Copyright (C) 2017 Lingqin Xia et al. This is an open access article distributed under the Creative Commons Attribution License, which permits unrestricted use, distribution, and reproduction in any medium, provided the original work is properly cited.

\begin{abstract}
Scanning microarc oxidation (SMAO) is a coating process which is based on conventional microarc oxidation (MAO). The key difference is that deposition in SMAO is achieved by using a stainless steel nozzle to spray an electrolyte stream on the substrate surface as opposed to immersing the workpiece in an electrolyzer. In the present study, SMAO discharge characteristics, coating morphology, and properties are analyzed and compared to results obtained from MAO under similar conditions. Results show that MAO and SMAO have comparable spark and microarc lifetimes and sizes, though significant differences in incubation time and discharge distribution were evident. Results also showed that the voltage and current density for MAO and SMAO demonstrate similar behavior but have markedly different transient and steady-state values. Results obtained from coating A356 aluminum sheet show that oxide thickness and growth rate in SMAO are strongly dependent on interelectrode spacing and travel speed. Analysis of the SMAO coating morphology and structure showed that a denser and slightly harder layer was deposited in comparison to $\mathrm{MAO}$ and is attributed to reduced porosity and increased formation of $\alpha-\mathrm{Al}_{2} \mathrm{O}_{3}$. Preliminary results indicate that SMAO represents a viable process for coating of aluminum surfaces.
\end{abstract}

\section{Introduction}

Microarc oxidation, also known as plasma electrolytic oxidation (PEO), is a plasma assisted surface coating process that was derived from conventional anodizing $[1,2]$ and has been the focus of much research activity in recent years. Due to the formation of a strong, adherent coating and minimal changes to the substrate properties, MAO is used to treat aluminum, magnesium, and titanium alloys to improve wear and corrosion resistance as well as to provide thermal and electrical barriers [3-8]. Due to its environmental friendliness and ability to form thick, dense oxide coatings composed of $\alpha-\mathrm{Al}_{2} \mathrm{O}_{3}$ and $\gamma-\mathrm{Al}_{2} \mathrm{O}_{3}$, MAO has also attracted increased attention as a potential replacement for hard anodizing [912]. While most attention has been focused on enhancing tribological properties, there has also been growing interest in using MAO as a means to deposit ceramic coatings on large aluminum panels in order to reduce noise and vibration in railway vehicles [13].

The typical MAO electrolyzer normally consists of an open topped tank constructed from stainless steel and contains a dilute electrolyte solution. A metal workpiece is immersed in the electrolyte bath in order for the surface to undergo electrochemical conversion and form a ceramic layer. However, in practice, this set-up results in several limitations. The first is that the maximum workpiece size that can be coated is largely governed by the dimensions of the electrolyzer. A second limitation on workpiece size is based on the power supply output as the maximum current density that can be applied will be a function of the total surface area being treated. A third limitation results from the need to immerse the anode in an electrolyte solution. As all exposed surfaces undergo conversion and form a uniform layer, this makes it complex to obtain controlled differentials in coating thickness. Furthermore, in order to selective coating, suitable masking must be applied to any surface or regions where modification is not desired.

Because comparable limitations also exist in anodizing, and as the primary difference results from the voltage level used, this suggests that comparable techniques might also be employed in MAO. One such example can be found in a method for locally repairing anodized parts that was 
developed by Khromov et al. and is described in Russian patent number RU2163272 [14]. Under this approach, a cylindrical workpiece is rotated at a peripheral speed of $0.5-1.0 \mathrm{~m} / \mathrm{min}$ and a localized electrolyte stream is sprayed on the surface to repair worn regions. To address the size limitations posed by the MAO electrolyzer, Pogrebnjak and Tyurin [15] used a similar method to apply coatings on the exterior surfaces of metallized graphite tubes. This set-up employed four circular cathodes which formed a chamber around a segment of the tube such that a pressurized electrolyte stream could be sprayed on the surface. The cathodes were arranged sequentially such that a stepped voltage was applied to the tube surface as it passed through the chamber to ensure smooth growth of the oxide layer. An alternative approach that can be used to coat prismatic and nonprismatic parts was proposed by Wen et al. [16]. Their method, which was termed spraying MAO or SMAO, involved replacing the electrolyzer tank with a portable nozzle. While Wen et al. demonstrated that the a SMAO set-up could be used to apply localized deposits on $2024 \mathrm{Al}$ alloy samples, due to the stationary nozzle configuration and manual set-up that was used, testing was restricted to a relatively narrow set of conditions. Furthermore, it must be considered that the use of a portable cathode introduces an additional set of parameters in the MAO process. As these parameters have not been considered in previous studies, it can be considered that further investigation of SMAO is warranted.

In the present study, an experimental set-up was developed and used to investigate the SMAO process. The study focused on analyzing SMAO discharge behavior, growth kinetics, coating morphology, and microhardness. Because different cathode and electrolyte flow configurations are used in SMAO and MAO, it was also of interest to determine how coating behavior was affected. To assess this, results obtained from a MAO set-up using comparable conditions are also presented and used as a baseline. While the use of a scanning or moving cathode has been previously proposed as a means for performing selective deposition [16], little testing has been conducted to date. Consequently, both stationary and moving SMAO cathodes were investigated and coating performance was assessed. As part of the assessment, interelectrode gap, polarity, and scanning speed were investigated and a summary of these effects is also provided.

\section{Experimental Set-Up and Procedure}

The experimental SMAO set-up used in the study was developed by modifying an existing MAO test stand. The primary modification consisted of connecting the output side of the electrolyte circulating pump to a threaded steel tube. A $15 \mathrm{~mm}$ diameter stainless steel nozzle was then attached to the free end of the tube and electrically connected to act as the cathode. For safety reasons and to eliminate the need for manual operation of the set-up, the tube/nozzle assembly was also mounted on a linear actuator (Figure 1). This enabled travel speed to be adjusted from $0 \mathrm{~mm} / \mathrm{s}$ to $15 \mathrm{~mm} / \mathrm{s}$ and provided the capability for the cathode to be used in either stationary or scanning mode such that localized spots or full surfaces could be coated on various sheet sizes. The linear

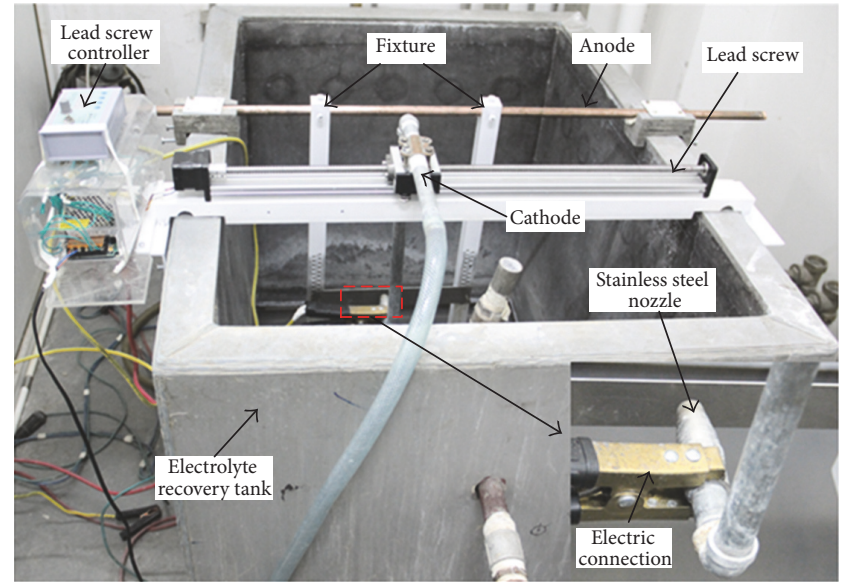

FIGURE 1: Photograph showing the SMAO set-up used in the study.

actuator was then attached to the top of an existing MAO electrolyzer which enabled it to function as both a reservoir and a recovery tank for the electrolyte solution.

To ensure that a consistent interelectrode gap and orientation was maintained between the cathode and the anode surface during each test, all samples were securely mounted in an adjustable fixture. The fixture was designed to accommodate different sheet sizes and was also repositionable such that the interelectrode gap could be adjusted from 0 to $500 \mathrm{~mm}$. Although various nozzle/workpiece orientations can be employed in SMAO, each workpiece was mounted vertically with the electrolyte stream being applied perpendicular to the surface. This orientation was used in part as Wen et al. [16] noted that it resulted in reduced microporosity in the SMAO coating.

As identical electrical sources can be used in SMAO and $\mathrm{MAO}$, an existing $75 \mathrm{~kW}$ power source with variable polarity, frequency, and current was employed and the main electrical parameters are summarized in Table 1.

For the investigation, $2 \mathrm{~mm}$ thick A356 aluminum alloy sheet was selected as the substrate material and the chemical composition is given in Table 2. To ensure that similar surface areas were treated in each test, the MAO samples of dimensions $10 \mathrm{~mm} \times 7 \mathrm{~mm} \times 2 \mathrm{~mm}$ were used. As the transverse ends of the MAO samples were masked by the support fixture, this resulted in an effective surface area of $180 \mathrm{~mm}^{2}$ and was equivalent to the transient discharging area of SMAO. Prior to be being coated, all specimens were sequentially ground using 400\#, 800\#, and 1200\# abrasive papers. Each specimen was then ultrasonically degreased in an acetone bath, rinsed in deionized water, and dried using a portable forced air dryer. A sodium silicate-based aqueous solution consisting of $\mathrm{NaOH}(2 \mathrm{~g} / \mathrm{L})$ and $\mathrm{Na}_{2} \mathrm{SiO}_{3}(10 \mathrm{~g} / \mathrm{L})$ was employed as the electrolyte for all SMAO and MAO tests. The electrolyte temperature was maintained within $20 \sim 40^{\circ} \mathrm{C}$ and a constant flow rate of $1.57 \times 10^{-4} \mathrm{~m}^{3} / \mathrm{s}$ was used in SMAO.

SMAO and MAO discharges processing was recorded using a FASTCAM Mini UX50 high speed camera (2.8 aperture, $5000 \mathrm{~Hz}$ frame rate, and $200 \mu$ s exposure) and a Canon EOS 60D digital camera (5.6 aperture and $12.5 \mathrm{~ms}$ 
TABLE 1: Summary of AC current and voltage parameters used in the investigation.

\begin{tabular}{lcccccccc}
\hline & \multicolumn{2}{c}{ AC1 } & \multicolumn{2}{c}{ AC2 } & \multicolumn{2}{c}{ AC3 } & \multicolumn{2}{c}{ AC4 } \\
& SMAO & MAO & SMAO & MAO & SMAO & MAO & SMAO & MAO \\
\hline Power rating/KW & 5 & 5 & 10 & 10 & 20 & 20 & 40 & 40 \\
Positive peak voltage/V & 700 & 594 & 710 & 656 & 725 & 703 & 731 & 750 \\
Negative peak voltage/V & -376 & -143 & -383 & -207 & -411 & -249 & -405 & -294 \\
Current/A & 4.3 & 6.7 & 4.5 & 18.4 & 4.62 & 31.6 & 4.73 & 41.4 \\
\hline
\end{tabular}

TABLE 2: Chemical composition of the A356 alloy.

\begin{tabular}{lcccccccc}
\hline Element & $\mathrm{Al}$ & $\mathrm{Si}$ & $\mathrm{Mg}$ & $\mathrm{Mn}$ & $\mathrm{Fe}$ & $\mathrm{Cu}$ & $\mathrm{Ti}$ & $P$ \\
\hline wt.\% & Balance & 7.23 & 0.332 & 0.001 & 0.112 & 0.001 & 0.128 & $<0.001$ \\
\hline
\end{tabular}

exposure). Prior to being coated, an aluminum sample was placed on a reference surface and the initial sample thickness, $H_{i}$, was measured using a micrometer. After being coated, the resulting total sample thickness, $H_{f}$, was then measured. The outward growth dimension, $T_{\text {out }}$, of the coating was obtained by taking the difference between $H_{f}$ and $H_{i}$. Inward growth was determined by first measuring the total coating thickness, $T$, using a ISOCOPE MPOR eddy current thickness meter. The inward growth dimension $T_{\text {in }}$ was then calculated by taking the difference between $T$ and $T_{\text {out }}$.

The surface and cross-sectional morphologies of the MAO and SMAO coatings were characterized using a CamScan 3400 scanning electron microscope (SEM). To analyze the phase composition of the as-deposited aluminum oxide layer, a Rigaku Smartlab X-ray diffraction diffractometer (XRD) ( $\mathrm{Cu} \mathrm{K} \alpha, 40 \mathrm{KV}, 40 \mathrm{~mA}$ ) was used. The as-deposited coating thickness on each specimen was measured using an ISOCOPE MPOR eddy current thickness meter and all values were reported as the average of 20 measurements. Coating hardness distribution in cross section was evaluated using an HMV-IT microhardness tester with a Vickers indenter under a $0.98 \mathrm{~N}(100 \mathrm{~g})$ load and a dwell time of $15 \mathrm{~s}$.

\section{Results and Discussion}

3.1. Discharge Behavior and Characteristics. When AC1 was used in SMAO, an incubation period of approximately $5 \sim 10 \mathrm{~s}$ was required for weak spark formation to occur after which the number of sparks began to increase quickly. After $10 \mathrm{~s} \mathrm{had}$ elapsed, sustained white spark discharges began to appear on the substrate surface in line with the impinging electrolyte stream as shown in Figure 2(a). The sparks were small and dense and formed a uniform, continuous membrane on the substrate surface that was approximately $19.5 \mathrm{~mm}$ in diameter. This value was slightly larger than the nozzle opening as some expansion of the pressurized electrolyte column occurred. After 2 minutes, the discharge distribution (Figure 2(b)) had expanded to a diameter of $29.7 \mathrm{~mm}$ and slightly yellowish microarc discharges began to appear at the center with an audible noise. Within 3 15 minutes, the diameter of the discharge area continued to expand from 33.9 to $37.8 \mathrm{~mm}$. During this time, microarc discharges continued to be prevalent in the center of discharge area while spark discharges were dominant in the surrounding periphery and coincided with the formation of thicker and thinner coatings, respectively. After 15 minutes, continued observation showed no further changes in either the size or composition of the spark and microarc discharge distribution and this was taken to indicate steady-state behavior.

For comparison purposes, identical AC power settings were also used in MAO. It was initially observed that no sparking occurred and that a number of gas bubbles formed on the workpiece surface and/or were released into the surrounding electrolyte. After an incubation period of $1 \sim 2 \mathrm{~s}$, a small number of white sparks appeared on the edges of workpiece and this was accompanied by increased formation of gas bubbles above the workpiece surface. This is consistent with previously reported observations for MAO $[17,18]$ and can be attributed to the edge effect of the electric field. After $5 \mathrm{~s}$, small, white sparks which tended to be weak and shortlived appeared randomly on the workpiece surface. With further treatment time, the spark discharge density increased and also began to propagate towards the center of each surface on the specimen. After 1 minute of operation, yellowish microarcs also began to develop across each surface and a low audible noise was emanated. Subsequent observations taken from photographs showed that the amount of spark discharges was reduced but that the number of microarc discharges had gradually increased. After 3 minutes had elapsed, the size of both discharges had noticeably increased while the densities had been gradually reduced. An additional observation was that some intermingling of sparks and microarcs was observed though the number of spark discharges tended to be prevalent on the inner region of the surface, whereas microarc discharges were more dominant on the outer periphery. Between 5 and 15 minutes, both sparks and microarc continued to be present and a loud, shrill audible noise could be heard. During 10 30 minutes, the microarcs were observed to have a degree of mobility and were able to change position while maintaining the shrill noise. After 40 minutes, no sparks were present and the size and life time of microarc discharges had further increased with a corresponding reduction in density. After $60 \mathrm{~min}-$ utes, only a few large, long-lived microarc discharges could 


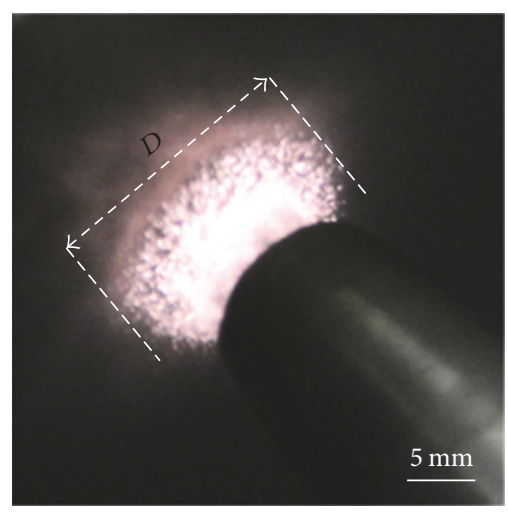

(a)

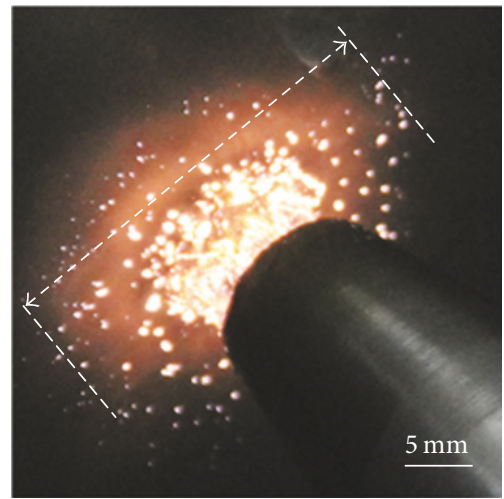

(d)

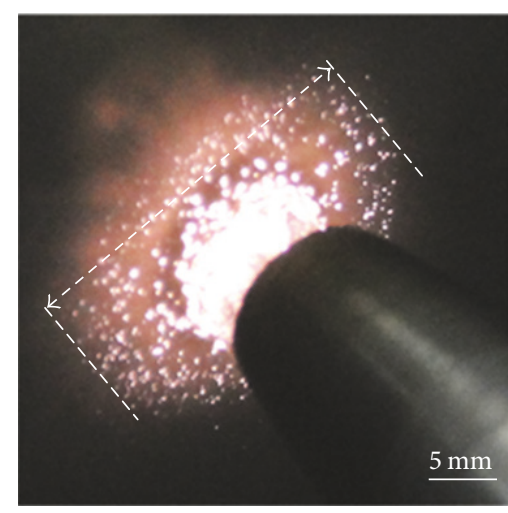

(b)

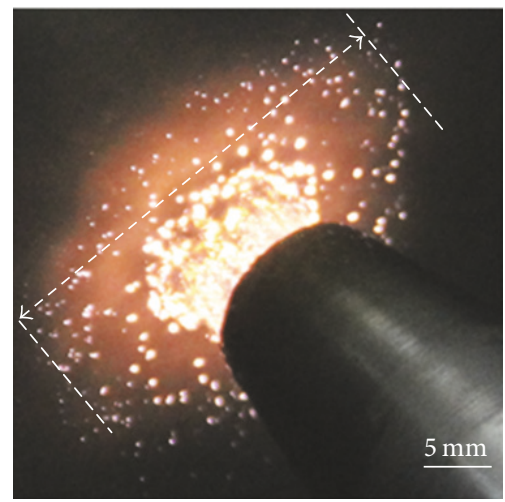

(e)

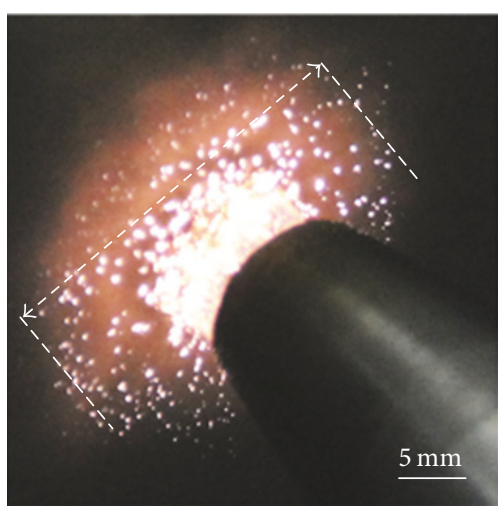

(c)

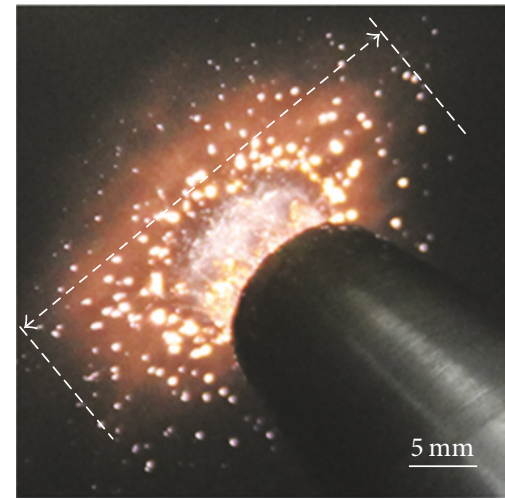

(f)

FIGURE 2: Photographs showing SMAO discharge distributions and diameters taken at (a) $10 \mathrm{~s}, D=19.5 \mathrm{~mm}$; (b) $2 \mathrm{~min}, D=29.7 \mathrm{~mm}$; (c) $3 \mathrm{~min}, D=33.9 \mathrm{~mm}$; (d) $5 \mathrm{~min}, D=35.7 \mathrm{~mm}$; (e) $10 \mathrm{~min}, D=36.9 \mathrm{~mm}$; (f) $15 \mathrm{~min}, D=37.8 \mathrm{~mm}$.

be observed and at this point coating thickness remained basically unchanged while surface roughness showed a slight increased.

Comparing SMAO and MAO discharge behavior in AC mode, the primary similarity is that sparks initially appeared on the cathode surface after which both processes tended to develop strong microarc discharges. For both SMAO and $\mathrm{MAO}$, the spark discharge duration increased gradually with treatment time (Figure 3(a)), while microarc lifetimes showed a gradual increase at first but demonstrated an abrupt increase after 30 40 minutes.

The primary differences in discharge behavior between SMAO and MAO can be summarized as follows. The first is that SMAO has a slightly longer incubation period than MAO due to the latter having a higher current density as seen in Table 1 for AC1. In contrast to MAO, no bubbles were evident in SMAO and this can be attributed to the continuous flow of the electrolyte stream. Based on high speed photography measurements, the diameter of the microarcs were found to be generally comparable and on the order of micrometers. However, while SMAO microarc size tended to increase over time, those for MAO showed a peak at 30 minutes after which values tended to decrease gradually. The most significant difference that can be noted is with respect to SMAO and MAO discharge behavior that can be seen in Figure 4. While the SMAO discharge area showed a gradual increase during the first 15 minutes of operation, the overall distribution of discharges tended to be consistent. Examining Figure 4(b), it can be seen that the distribution of SMAO discharges can generally be divided into three concentric regions characterized by different densities. Region I corresponds to the impinging electrolyte stream and is evidenced by a dense, discharge intensive area. Region II represents a transition zone which is characterized by a sparser distribution of intermediate sized discharges. Region III is the outermost ring and contains a moderate density of smaller discharges.

Some insight about the SMAO discharge geometry can be obtained by considering electrical discharge machining (EDM). In both MAO and EDM, it can be considered that multiple spark discharges occur in a dielectric medium and superheated bubbles are formed. Prior EDM research [1923] has shown that the heat flux of a single spark discharge event can be satisfactorily represented using a Gaussian distribution. It is also well established from plasma physics that the thermal flux of an arc can also be represented using a Gaussian distribution. Given the large number of spark and microarc discharges that simultaneously exist on the SMAO anode surface and symmetric distribution, the aggregate thermal effect can be taken as the summation of the individual heat fluxes which in turn will be a Gaussian distribution. 


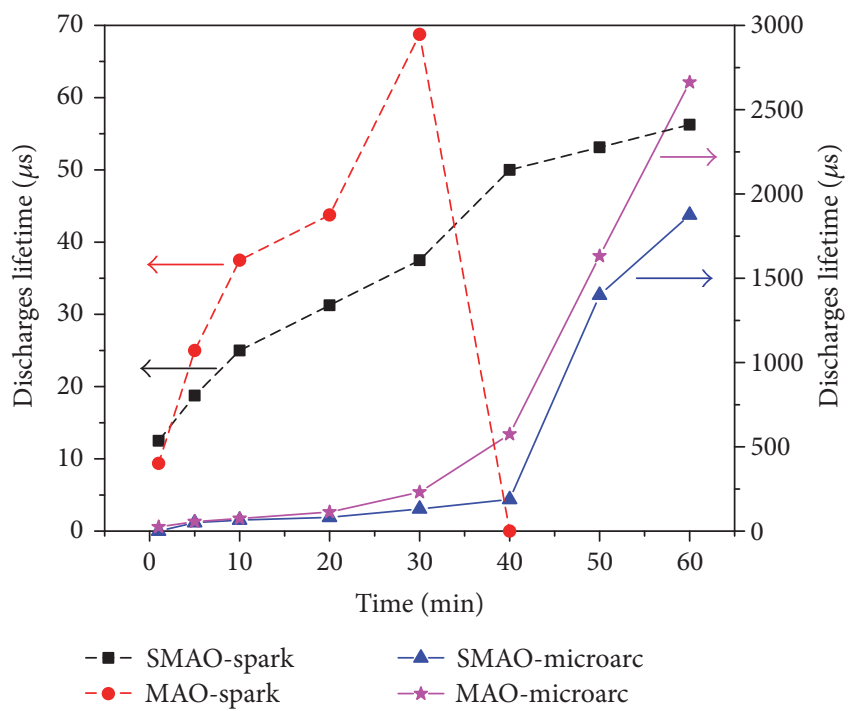

(a)

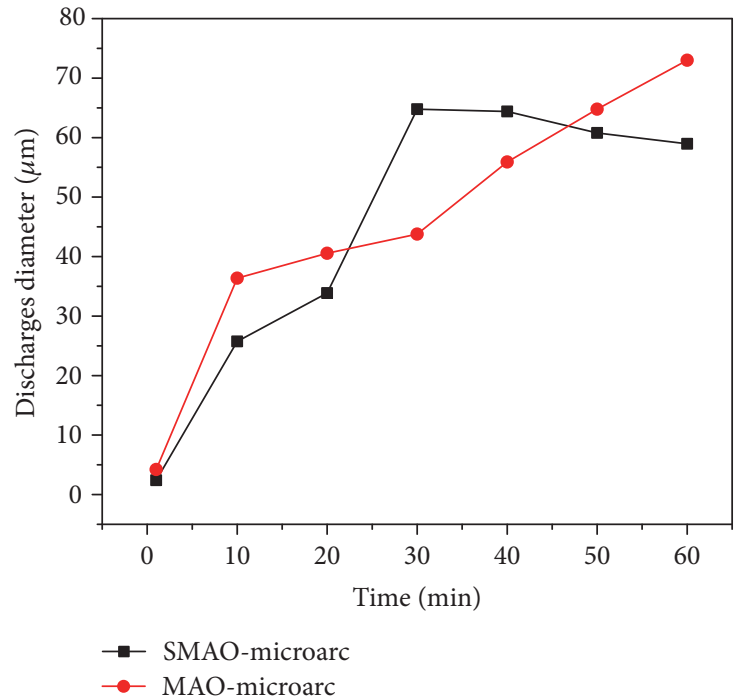

(b)

Figure 3: MAO and SMAO (a) discharge life time-treatment time curves and (b) discharge diameter-treatment time curves for conventional and SMAO processing.

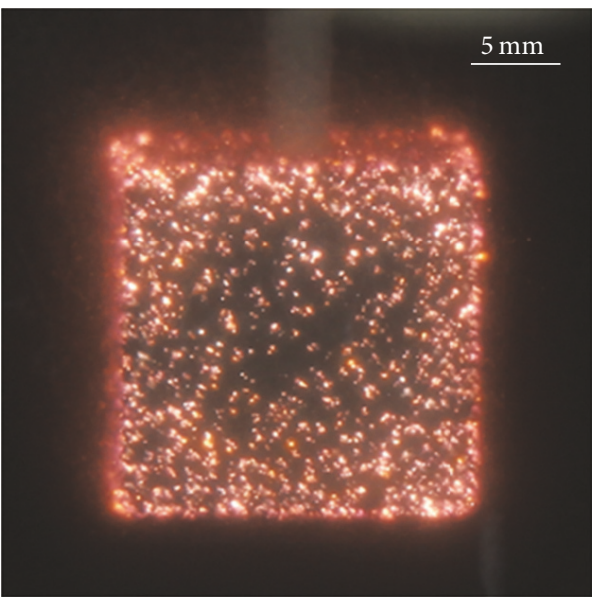

(a)

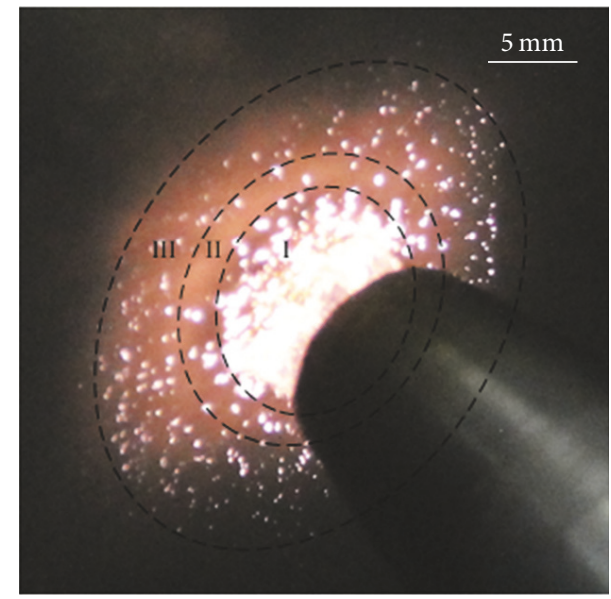

(b)

Figure 4: Comparison of spark discharges in (a) MAO and (b) SMAO after 2 min of treatment.

Some evidence to support this was obtained by measuring the variation in coating thickness across the diameter of an SMAO deposit. This is shown in Figure 5 where it can be seen that, after 5 and 60 minutes, the minimum coating thickness is found on the periphery (Region III), while the maximum thickness occurs in the center (Region I) in both cases.

3.2. Voltage-Time Response during SMAO and MAO. The voltage-time response curves recorded at $\mathrm{ACl}$ are shown in Figure 6 for SMAO and MAO. For SMAO, both 5 and $10 \mathrm{~mm}$ interelectrode gaps were considered. As can be seen in Figure 6, the SMAO and MAO voltage-time responses demonstrate similar 3-stage behavior corresponding to incubation, breakdown, and steady-state behavior. In addition to difference in steady-state voltage values, notable differences were also observed during the incubation period and breakdown and these can be seen in Figure 7. During stage I, the voltage for all three cases shows a steep increase corresponding to formation of an anodic coating layer in Figure 7 but demonstrates markedly different breakdown values which mark the first spark appearance on the workpiece surface. It can be seen that, at the end of stage I, the SMAO breakdown voltages of 439 and $500 \mathrm{~V}$ were proportional to the interelectrode gap but were substantially lower than the $560 \mathrm{~V}$ value observed for MAO. This is consistent with the fact that stronger electric fields tend to develop at shorter interelectrode gaps. Differences can also be seen in stage II (Figure 7) where the SMAO voltages show a steady, gradual increase while the voltagetime response of MAO demonstrated an upper $(605 \mathrm{~V})$ and lower voltage plateau $(560 \mathrm{~V})$. Finally, during stage III, which 


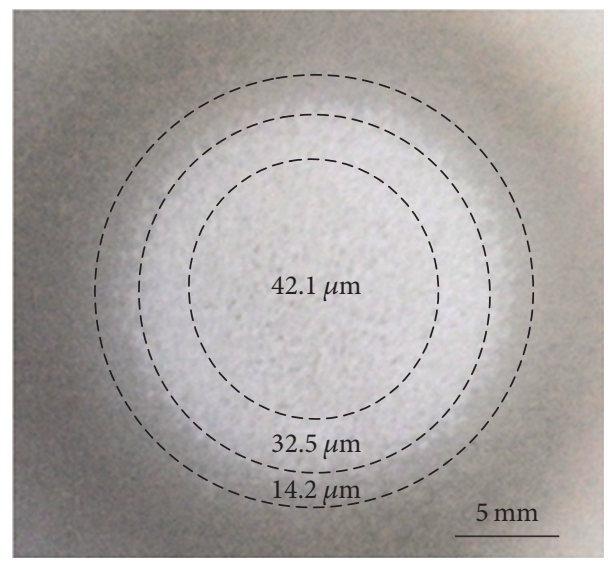

(a)

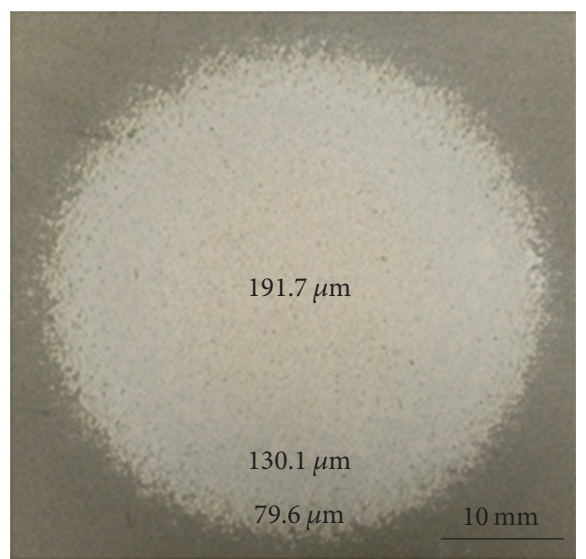

(b)

FIGURE 5: Photographs showing thickness variation in an SMAO coating deposited on an A356 aluminum substrate using AC1 and 5 mm interelectrode gap after (a) $5 \mathrm{~min}$ and (b) $60 \mathrm{~min}$ of treatment.

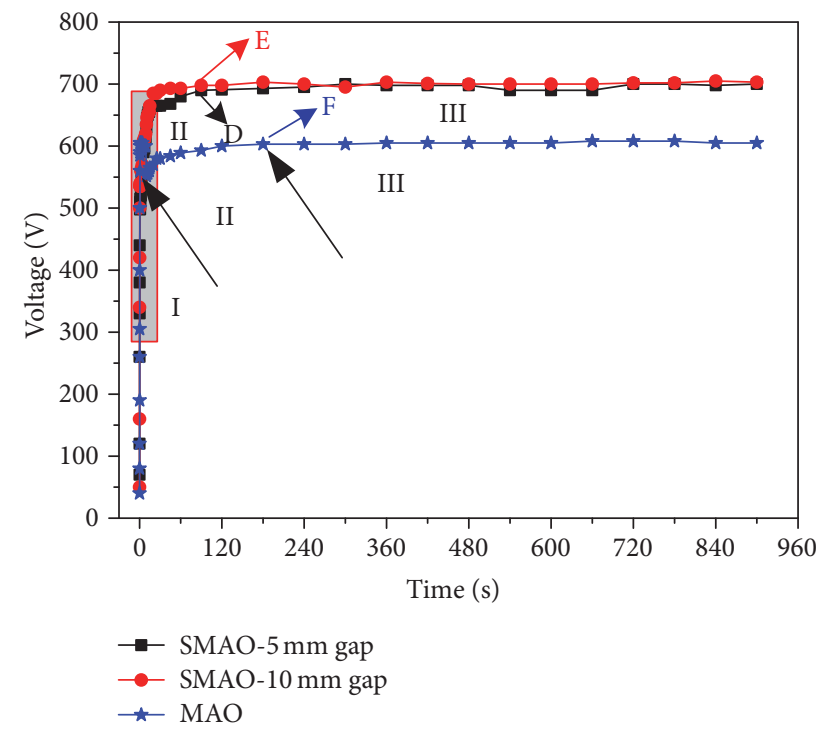

FIGURE 6: Long-term voltage-time response curves for SMAO ( $5 \mathrm{~mm}$ and $10 \mathrm{~mm}$ interelectrode gap and stationary anode) and MAO for A356 alloy.

represents steady-state behavior and formation of microarc discharges, it is evident from Figure 6 that the MAO steadystate voltage is almost $100 \mathrm{~V}$ lower than the corresponding SMAO values which were on the order of $600 \mathrm{~V}$ and 690 $700 \mathrm{~V}$, respectively.

3.3. Effect of SMAO Process Parameters on A356. While it has generally been considered that the effect of interelectrode spacing is negligible in MAO, work published by researchers such as Wei et al. [24] has suggested that it does influence MAO current and oxidation efficiency. To determine if there were similar effects on SMAO discharge performance and coating kinetics, a series of tests were run using a stationary cathode and interelectrode gaps ranging from 0 to $30 \mathrm{~mm}$. Testing was conducted at both $\mathrm{ACl}$ and $\mathrm{AC} 2$ power levels for

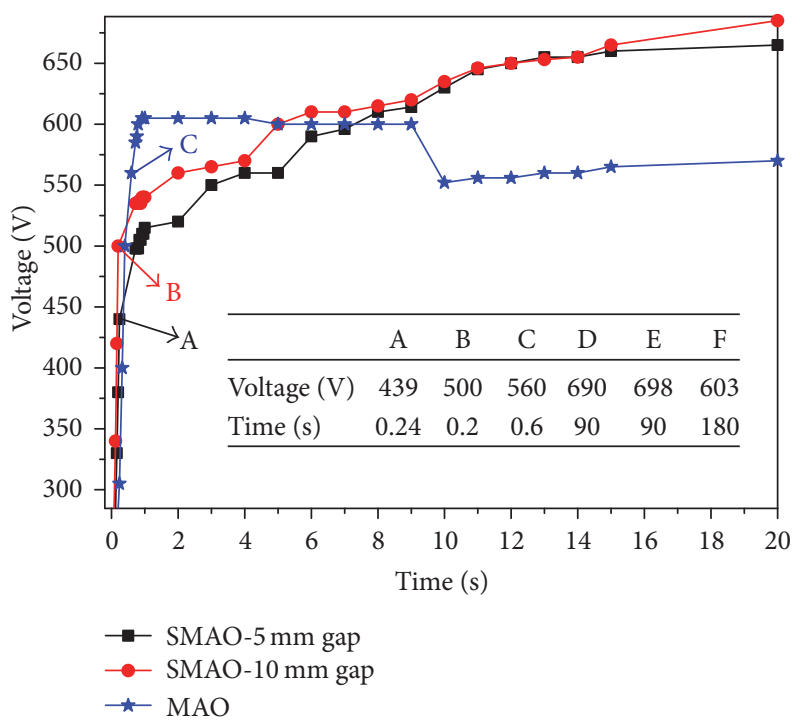

FIgURE 7: Transient voltage-time response curves for SMAO (5 and $10 \mathrm{~mm}$ interelectrode gaps and stationary anode) and MAO for A356 alloy.

a total of 900 seconds. MAO test results were also obtained using the same power settings for comparison. Coating thickness was also measured at the center of each SMAO deposit at 60 -second intervals.

SMAO incubation times were recorded and the results in Table 3 clearly show that it is proportional to the interelectrode gap at distances of $5 \mathrm{~mm}$ and greater. Tests were also conducted using interelectrode gaps less than $5 \mathrm{~mm}$, but this resulted in the tip of the stainless steel nozzle becoming heavily oxidized and eroded. This was caused by intense arcing and high temperatures which resulted from breakdown of the air column surrounding the electrolyte stream. Consequently, all gaps less than $5 \mathrm{~mm}$ were considered to be impractical for further use in the SMAO setup because the oxygen and hydrogen byproducts generated 
TABLE 3: Summary of SMAO incubation times obtained for different interelectrode gaps testing.

\begin{tabular}{lllllc}
\hline Interelectrode gap $/ \mathrm{mm}$ & 5 & 10 & 15 & 25 & $>30$ \\
Incubation time/s & 5 & 15 & 28 & 40 & No spark \\
\hline
\end{tabular}

during the SMAO process cannot separate efficiently and this likely causes breakdown of the passive film on the stainless steel nozzle. When interelectrode gaps of 5 and $10 \mathrm{~mm}$ were used, continuous and steady discharges were observed and a uniform coating formed on the substrate surface in both cases. When the interelectrode gap was increased to $15 \mathrm{~mm}$, this resulted in a reduced discharge intensity and a smaller area of oxide deposit formed. Similar behavior resulted at a $25 \mathrm{~mm}$ interelectrode gap. This can be attributed to the fact that an increased interelectrode gap will result in decreased electric field strength at the surface of the anode. At interelectrode distances of $30 \mathrm{~mm}$ and greater, the electrolyte stream became too dispersed due to gravitational effects and no spark formation was observed at the anode. Based on these results, it was determined that an interelectrode gap between 5 and $10 \mathrm{~mm}$ represented an optimal range for the SMAO setup used in the study.

Current density as a function of time was also recorded at $\mathrm{ACl}$ for SMAO (5 and $10 \mathrm{~mm}$ interelectrode gaps) and for MAO and these curves are shown in Figure 8. It can be seen from Figure 8(a) that both processes demonstrate similar trends and characteristics though it is evident that the current density for MAO is significantly higher and has a more pronounced slope. The maximum current density in SMAO for the 5 and $10 \mathrm{~mm}$ interelectrode gaps was $73.3 \mathrm{~A} / \mathrm{dm}^{2}$ and $41.7 \mathrm{~A} / \mathrm{dm}^{2}$, respectively, whereas the corresponding value for MAO was $111.7 \mathrm{~A} / \mathrm{dm}^{2}$. Examination of Figure 8(b) also shows that once the current density achieved its maximum value at $0.3 \mathrm{~s}$, it showed a significant drop in all three cases after which current density underwent a more gradual decrease as an oxide layer began to develop on the sample surface. The gradual decrease in current density is reflective of oxide layer formation and the larger current density and slope in MAO indicate that the initial layer formed is thicker and has a higher growth rate than SMAO.

As previous results indicate that the interelectrode gap does influence the incubation period and current density in SMAO, it was also of interest to study the effect on coating thickness and growth rate. These results are shown in Figure 9 where the data clearly shows that SMAO coating thickness is inversely proportional to the interelectrode gap and is inversely proportional to anode/cathode separation. It can be seen from Figure 9 that, after a total treatment time of 900 seconds, an average coating thickness of $108.95 \mu \mathrm{m}$ was obtained using the $5 \mathrm{~mm}$ gap, whereas an average thickness of $41.1 \mu \mathrm{m}$ resulted for the $10 \mathrm{~mm}$ gap setting. Furthermore, SMAO coating growth rate was not linear and tended to follow a two-stage growth rate at both the 5 and $10 \mathrm{~mm}$ interelectrode gap settings. The first stage corresponds to anodic oxidation while the second stage consists of microarc oxidation which is evidenced by the presence of small white specks in the ceramic coating. The length of stage 1 also

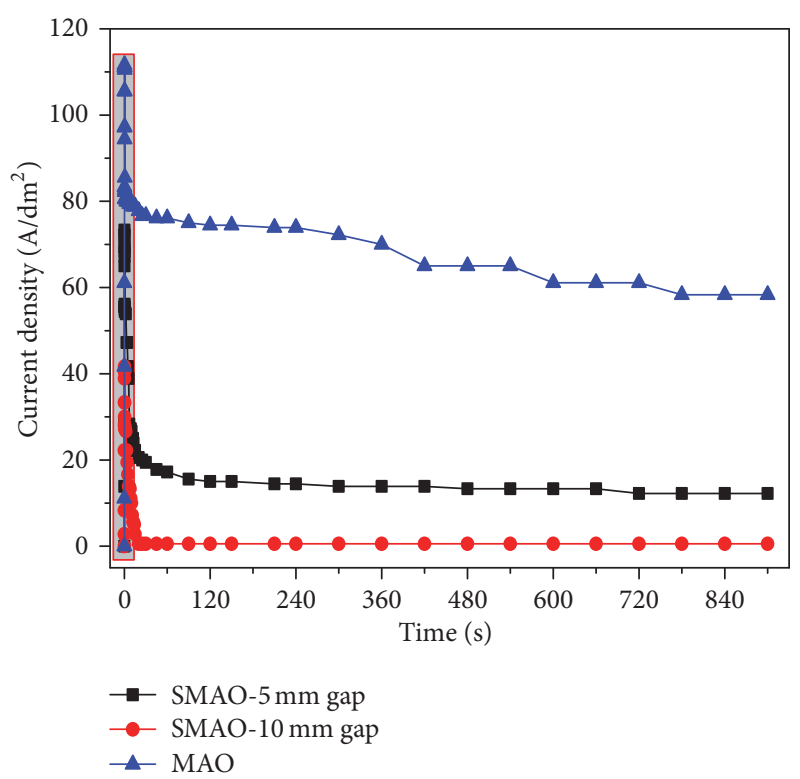

(a)

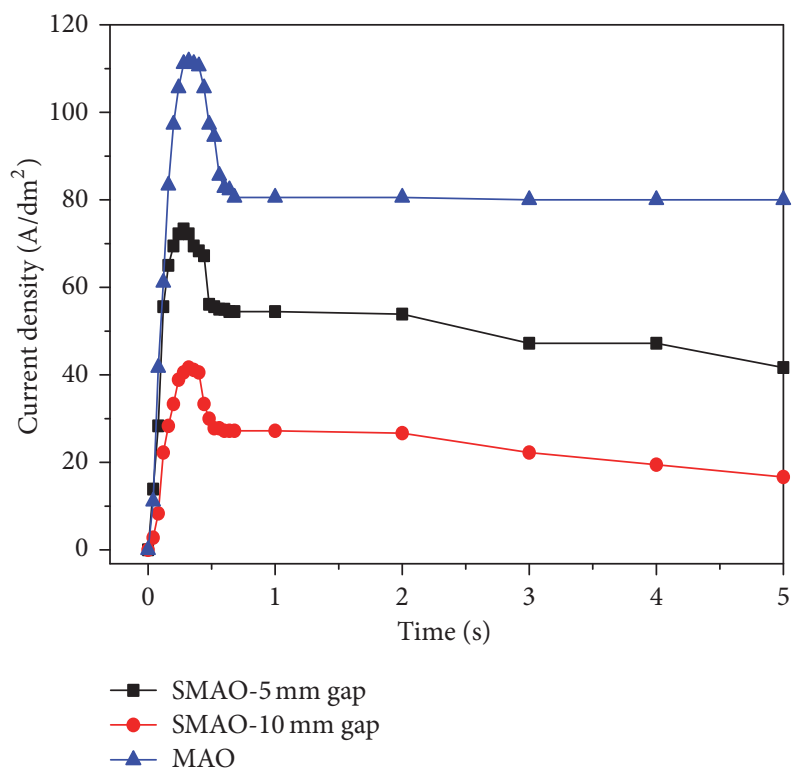

(b)

FIgURE 8: Current density-time responses of SMAO and MAO for A356 alloy based on ACl setting showing (a) long-term and (b) transient behavior.

differed with interelectrode gap in that the second stage began after $3 \mathrm{~min}$ for the $5 \mathrm{~mm}$ gap, whereas it was delayed until $8 \mathrm{~min}$ at the longer mm gap. It is also apparent from the slope of each curve in Figure 9 that the shorter gap also resulted in a SMAO higher deposition rate with an average growth rate of $0.121 \mu \mathrm{m} / \mathrm{s}$ at $5 \mathrm{~mm}$ as compared to $0.046 \mu \mathrm{m} / \mathrm{s}$ at $10 \mathrm{~mm}$.

Comparing SMAO and MAO growth rates, it can be seen that these were generally comparable for treatment times of $180 \mathrm{~s}$ or less. However, for treatment times in excess of 180 seconds, the MAO growth rate can be seen to increase sharply and is able to produce a significantly thicker coating. This is in agreement with results obtained by Wen et al. [15] 


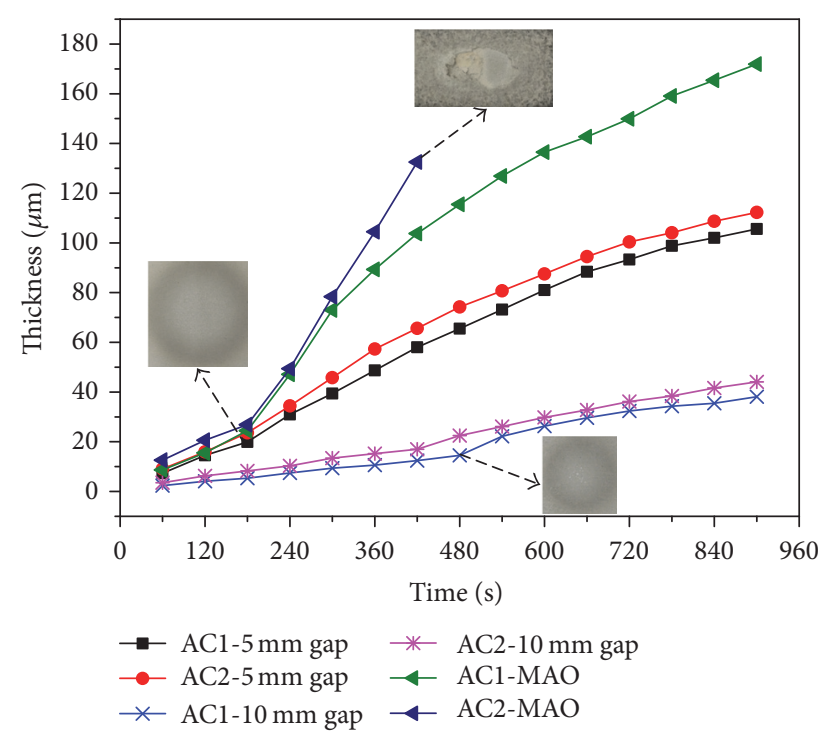

FIGURE 9: SMAO and MAO oxide layer thickness as a function of total treatment time using a $\mathrm{Na}_{2} \mathrm{SiO}_{3} / \mathrm{NaOH}$ electrolyte solution.

who also found that MAO tended to provide thicker coatings at higher deposition rates. The most likely reason for the large difference in deposition rates observed in Figure 9 is that the current density varied with the interelectrode gap and process used. Examining Figure 8(a), it can be seen that the current density at the $5 \mathrm{~mm}$ interelectrode gap and for MAO is significantly higher than for $10 \mathrm{~mm}$ gap. While further investigation is needed, based on the results obtained, they demonstrate that coating deposition rate is influenced markedly by both time and interelectrode distance.

As expected, higher power settings consistently resulted in increased coating thickness for all conditions tested. However, based on the results shown in Figure 9, it can also be seen that the power setting used also had markedly different effects in SMAO and MAO. Here too, the result is also likely due to the differences in current density produced at each condition. In comparison to MAO, SMAO current density and coating thickness appear to be much less sensitive to the power setting that was used for each interelectrode gap. Based on the curves shown in Figure 9, the maximum difference in coating thickness between $\mathrm{AC} 1$ and $\mathrm{AC} 2$ for SMAO is only $8.7 \mu \mathrm{m}$, while a much larger spread of $28.7 \mu \mathrm{m}$ was observed when the same settings were used for MAO.

3.4. Travel Speed. Because coating formation and growth are based in part on temperature and diffusion, it was hypothesized that the thickness of the SMAO deposit would be inversely proportional to the travel speed. To verify this, the interelectrode gap was set to $5 \mathrm{~mm}$ and cathode travel was varied using seven different speeds ranging from 0.6 to $15 \mathrm{~mm} / \mathrm{min}$ at $\mathrm{ACl}$ and $\mathrm{AC} 2$. The resulting thicknesses are shown in Figure 10 and confirm that coating thickness is inversely proportional to travel speed over the range of travel speeds considered and can be represented using an exponential decay function. The cross-sectional average

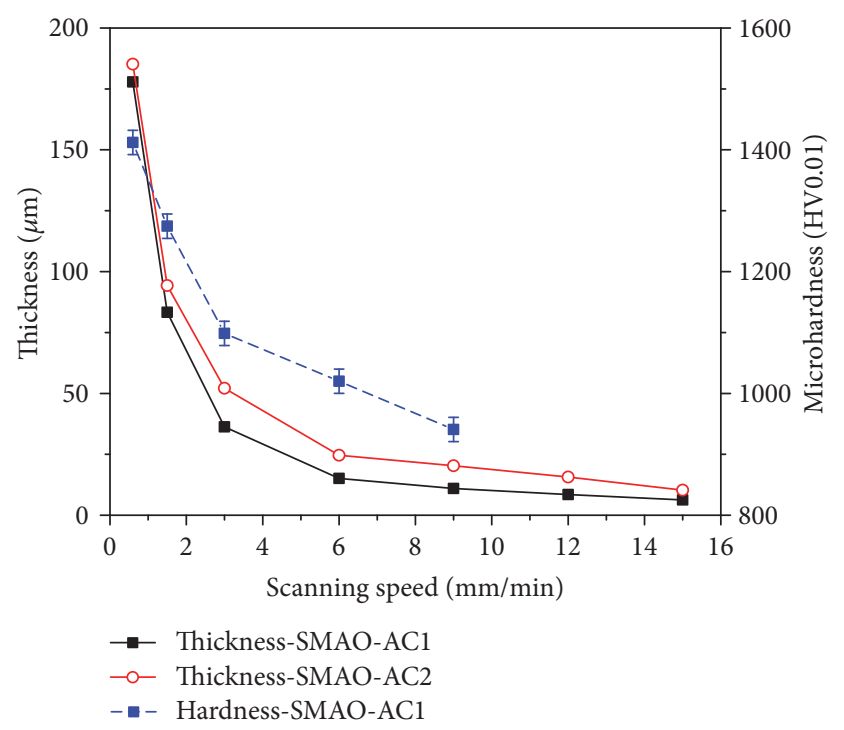

FIgURE 10: Plot showing coating thickness as a function of cathode travel speed for A356 alloy using a $\mathrm{Na}_{2} \mathrm{SiO}_{3}$ and $\mathrm{NaOH}$ electrolyte solution ( $\mathrm{ACl}$ and $5 \mathrm{~mm}$ interelectrode gap).

microhardness measurements for deposits made using AC1 were also recorded and can be seen to follow the same trend in Figure 10. While further testing is warranted to optimize deposition rate and coating properties, the results do confirm that it is possible to deposit an oxide layer using a moving cathode.

3.5. Microhardness. In addition to studying and comparing discharge behavior, it was also of interest to analyze the hardness of the as-deposited SMAO coatings. While identical power settings $(\mathrm{ACl})$ were applied, treatment times of 13 and 7 minutes were used for SMAO $(5 \mathrm{~mm}$ interelectrode gap, stationary nozzle) and $\mathrm{MAO}$, respectively, to ensure that a $100 \mu \mathrm{m}$ thick coating was achieved in both cases. After MAO and SMAO treatments had been performed, microhardness surveys were then taken over the coating cross sections. As a baseline, the microhardness of the A356 sheet was measured prior to coating and was found to be $80.6 \mathrm{HV}$. The average hardness results for both conditions are shown in Figure 11 and it can be seen that similar hardness gradients developed though SMAO tended to yield a slightly harder coating $(1500 \mathrm{HV})$ in comparison to MAO $(1300 \mathrm{HV})$. In both SMAO and MAO, the maximum (peak hardness) occurred at a distance of $10 \mu \mathrm{m}$ from the interface and gradually decreased thereafter to a minimum value of 959 and $788.6 \mathrm{HV}$, respectively, near the coating surface.

The increased SMAO coating hardness can be attributed in part to a higher $\alpha-\mathrm{Al}_{2} \mathrm{O}_{3}$ phase content which was evidenced by XRD analyses taken at the top surface of both samples. The results are shown in Figure 12 and the XRD spectra show that similar characteristic spectra and peak structures are present and that some mullite was also present in both coatings due to the presence of silica in the electrolyte. However, it can be seen from the XRD spectra that the peak intensity of the $\alpha-\mathrm{Al}_{2} \mathrm{O}_{3}$ phase is slightly greater in SMAO 


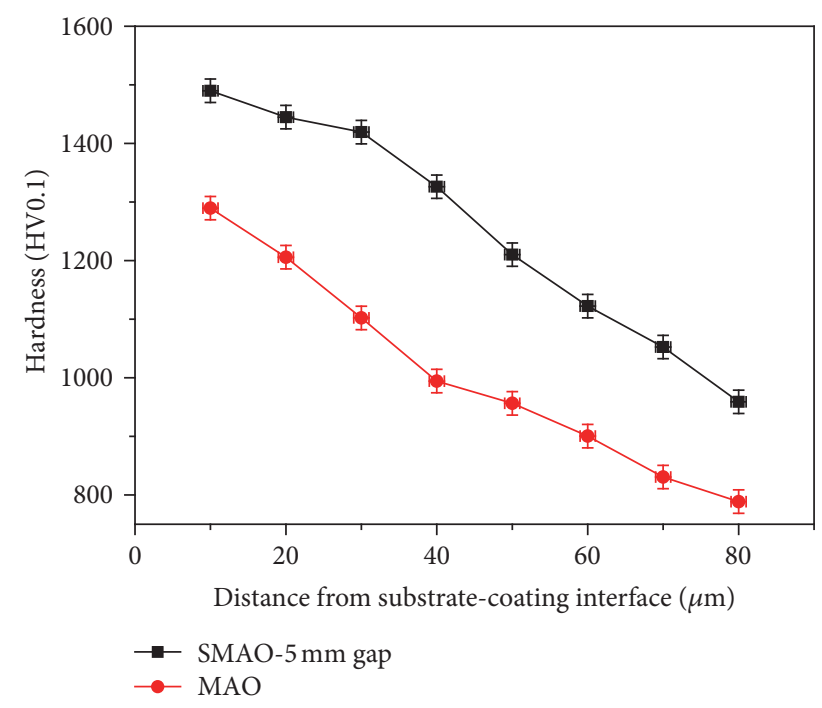

FIgURE 11: Vickers hardness profiles of SMAO and MAO deposits.

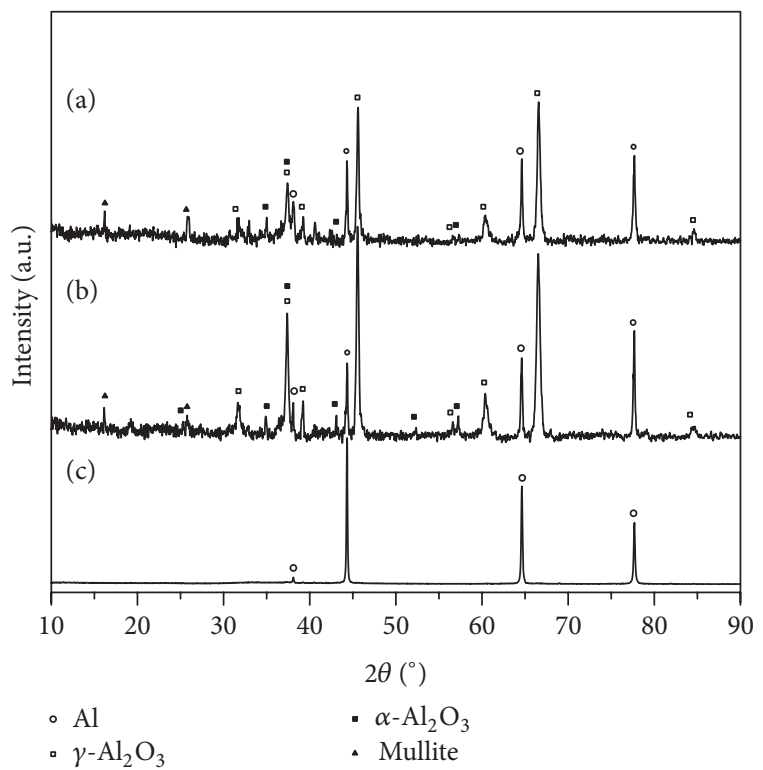

FIgURE 12: XRD patterns of coatings deposited at AC1 using a $\mathrm{Na}_{2} \mathrm{SiO}_{3}+\mathrm{NaOH}$ electrolyte and $\mathrm{A} 356$ alloy substrate for (a) MAO and (b) SMAO and (c) A356 alloy substrate.

than in MAO. This indicates a higher $\alpha-\mathrm{Al}_{2} \mathrm{O}_{3}$ content and is consistent with the microhardness results shown in Figure 11.

While the literature regarding $\alpha-\mathrm{Al}_{2} \mathrm{O}_{3}$ formation in $\mathrm{MAO}$ is not entirely unanimous, some observations can be made with respect to the different levels observed in the MAO and SMAO coatings. It has been previously noted that the accumulation of thermal energy in thicker coatings can help to generate the higher temperatures required to promote the $\gamma \rightarrow \alpha-\mathrm{Al}_{2} \mathrm{O}_{3}$ phase transition [25-27]. However, as phase changes normally require atomic rearrangement, the effect of time also needs to be considered. With respect to conditions in SMAO, it can be hypothesized that while the coatings tend to be thinner than those in MAO, and as such would favor formation of $\gamma-\mathrm{Al}_{2} \mathrm{O}_{3}$ phase, the sustained high density of discharges occurring in SMAO will likely result in sufficient temperature and time conditions that would promote the $\gamma \rightarrow \alpha-\mathrm{Al}_{2} \mathrm{O}_{3}$ phase transition.

To further analyze the difference in SMAO and MAO microhardness results, the cross-sectional morphologies from both samples were analyzed using SEM. It can be seen from the SEM micrographs in Figure 13 that a characteristic MAO coating consisting of a looser surface layer and a more compact interior layer formed in both cases. Furthermore it can be seen in both micrographs that the coating surface is irregular and a wavy metal-oxide interface having strong adhesion is present. However, when a comparison of the outer and subsurface layers of the MAO and SMAO deposits shown in Figures 13(a) and 13(b) is made, it is evident that the SMAO deposit is less porous which would also support the higher microhardness values that were observed. The increased density of the SMAO surface layer will provide improved tribological performance.

3.6. Coating Formation and Growth Kinetics. It is well established that MAO oxide layers develop by simultaneous inward and outward growth emanating from the original substrate surface. To confirm this behavior in SMAO, the inward and outward growth rates of the aluminum oxide layer were characterized using $\mathrm{ACl}$, a stationary nozzle, and $5 \mathrm{~mm}$ interelectrode gap.

The total thickness $T_{\text {total }}$, inward growth thickness $T_{\text {in }}$, and outward growth thickness $T_{\text {out }}$ of the SMAO coating were measured as a function of treatment time and the results are shown in Figure 14. Based on the behavior observed for SMAO, it can be seen that it is quite consistent with results that have been reported for MAO using aluminum [28]. The total coating thickness can be seen to follow a bilinear curve where the higher growth rate occurred during the first 30 minutes. It can also be seen that coating growth over the range of processing time that was considered is primarily due to outward growth of the layer. Furthermore, the bilinear characteristics of coating growth are mainly due to outward growth. In comparison, the inward growth rate was lower and tended to be relatively constant. Although there are differences in growth rate, based on the similarities in simultaneous inward/outward growth patterns, this suggests that the coating growth mechanism is the same in SMAO and MAO. As the growth mechanism has been described elsewhere [29, 30], it will not be repeated here. Further support that similar growth mechanisms are involved can be seen in Figure 13 where the coatings for both processes demonstrate characteristic artifacts that include discharge columns and porosity.

3.7. Surface Morphology. The surface morphology of the MAO and SMAO oxide coatings obtained after 15 minutes of treatment performed at $\mathrm{ACl}$ is shown in Figure 15. The morphologies of the SMAO and MAO coatings in Figures 15(a) and 15(d), respectively, exhibit both the characteristic MAO structure with numerous discharge channels and evident volcano-like surface features [31, 32]. However, it can 


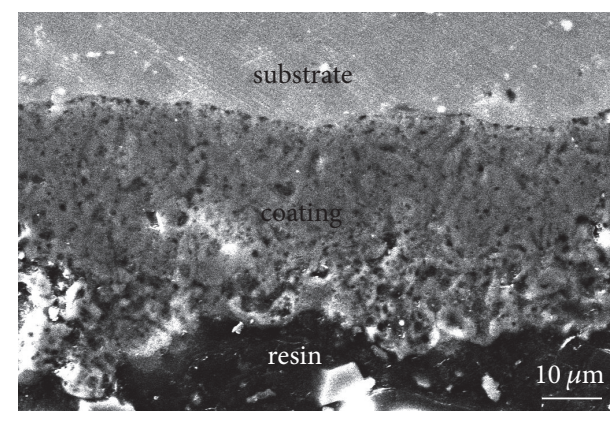

(a)

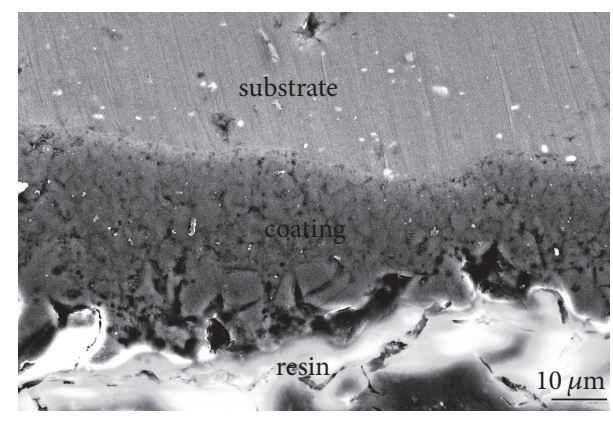

(b)

FIGURE 13: SEM micrographs showing cross sections of (a) MAO and (b) SMAO oxide deposits.

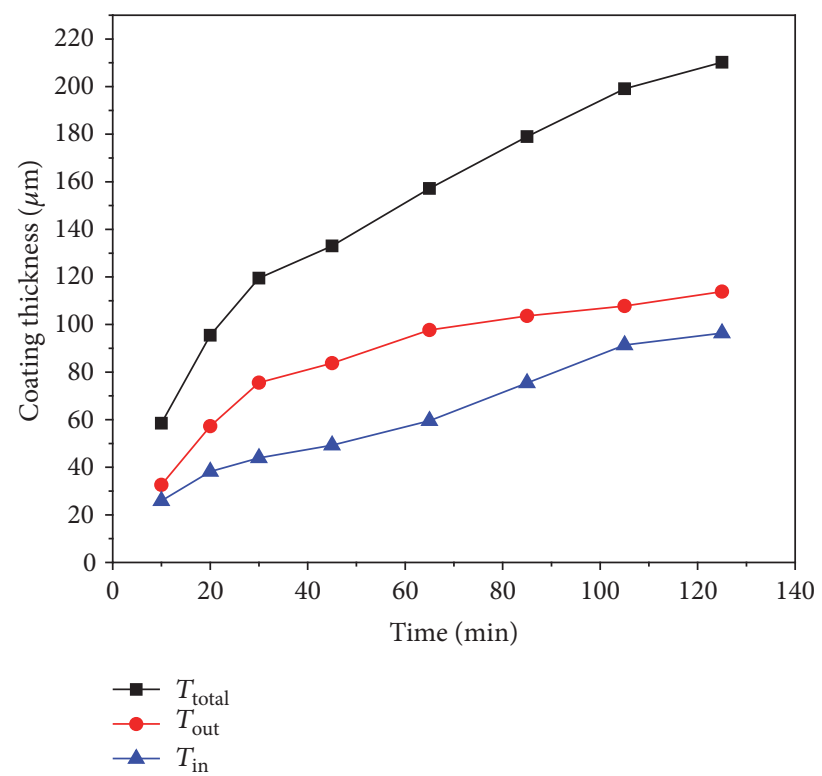

FIGURE 14: Curves showing total, outward, and inward growth behavior of the SMAO coating as a function of time on A356 substrate at $\mathrm{ACl}$, interelectrode gap $5 \mathrm{~mm}$, and stationary nozzle using a $\mathrm{Na}_{2} \mathrm{SiO}_{3}+\mathrm{NaOH}$ electrolyte stream.

be seen that the SMAO surface has a higher density of small discharge channels (Figure 15(b)) which varied within 0.1 $3 \mu \mathrm{m}$. In comparison, the pore diameters of MAO coating were larger and ranged within $2 \sim 6 \mu \mathrm{m}$ (Figure 15(e)). This is consistent with the size differentials between the SMAO and MAO discharges reported in Figure 3(b) and suggests that a smoother surface resulted. This feature is the result of higher current density passed through MAO samples as well as an increase in the rate of oxide layer formation [33].

The difference of the surface morphology caused by SMAO and MAO process can also be attributed to the difference of current density in the SMAO and MAO processes. It is known that a higher current density will produce a larger pore size. This is because a higher current density will increase the energy density and enhance the plasma and produce a larger pore size.

\section{Conclusions}

In the present study, an experimental SMAO set-up was developed and used to investigate discharge behavior and coating kinetics on $2 \mathrm{~mm}$ thick A356 aluminum sheet using a stationary cathode. A comparison was also made using results obtained from MAO under similar processing conditions. Coating thickness obtained using a moving cathode was also considered and analyzed. Future work will include a more detailed analysis of SMAO spark discharge characteristics as well as performing more comprehensive characterization and testing of coating properties. SMAO coating of alloys such as titanium, magnesium, and additional aluminum alloys will also be considered. Based on the results obtained from the study, the following conclusions can be made:

(1) MAO provides higher coating deposition rates than SMAO due to the larger current density that resulted at each power setting used.

(2) Discharge behavior and coating growth rate in SMAO are both sensitive to the interelectrode gap. For the SMAO set-up that was used, the optimal interelectrode gap was found to be between 5 and $10 \mathrm{~mm}$.

(3) A harder and a denser coating can be achieved in SMAO in comparison to MAO under identical processing conditions. This is attributed to the reduced porosity present in the subsurface and surface regions as well as slightly increased levels of $\alpha-\mathrm{Al}_{2} \mathrm{O}_{3}$ in the SMAO coating.

(4) Coating growth in SMAO is proportional to time and follows the same inward-outward growth pattern typically exhibited in MAO. Outward growth is dominant though comparable outward and inward growth rates were observed at extended treatment times.

(5) Coating thickness in SMAO is inversely proportional to travel speed when a moving cathode is used.

\section{Conflicts of Interest}

The authors declare that they have no conflicts of interest. 


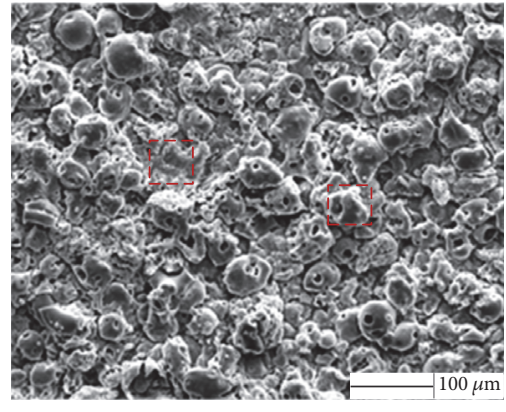

(a)

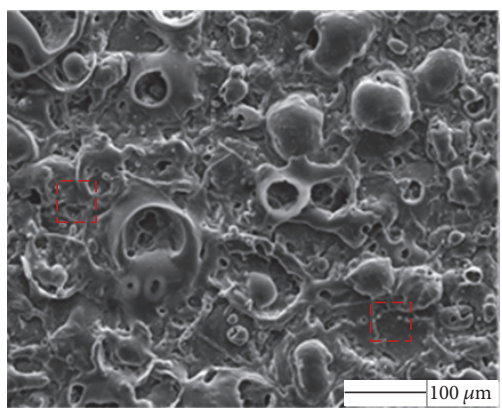

(d)

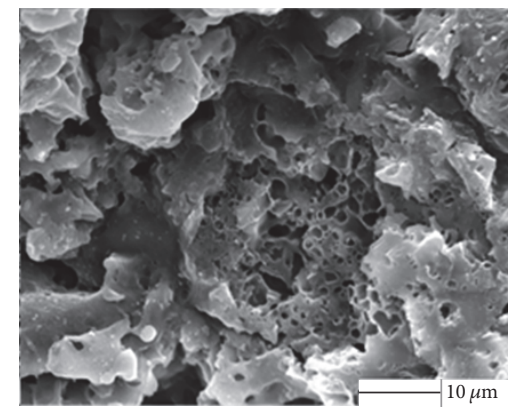

(b)

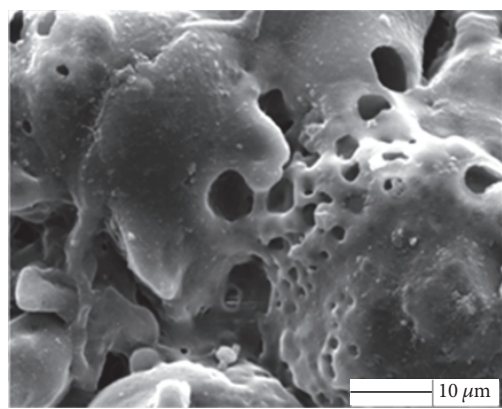

(e)

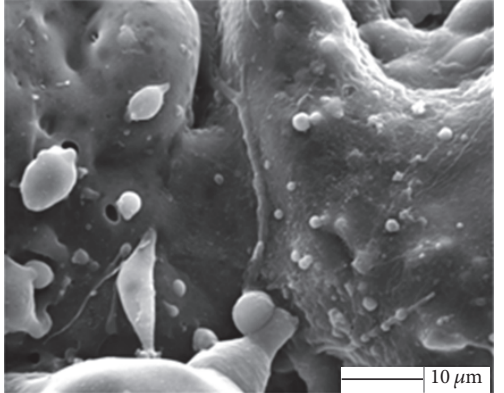

(c)

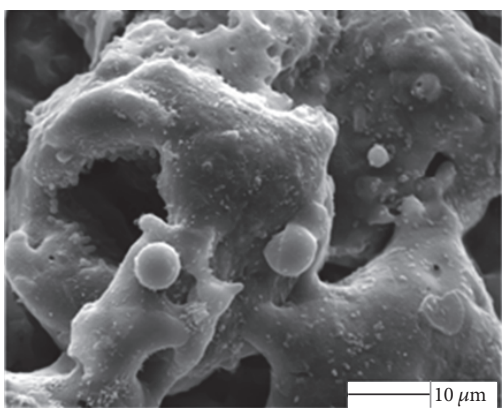

(f)

FIGURE 15: Surface morphology of the A356 alloy after $15 \mathrm{~min}$ : (a) and (d) are, respectively, SMAO (interelectrode gap $5 \mathrm{~mm}$, zero velocity) and MAO; (b) and (c) are the high magnification of the dotted rectangle area; (e) and (f) are the high magnification of the dotted rectangle area.

\section{Acknowledgments}

The authors would like to acknowledge support from the National Science Foundation of China (no. 51371022) and the Central College Basic Scientific Research Business Expenses Special Funds (2015JBM076) for conducting this work.

\section{References}

[1] S. Wernick, R. Pinner, and P. G. Sheasby, The surface treatment and finishing of aluminium and its alloys, ASM International, Teddington, London, UK, 5th edition, 1987.

[2] V. F. Henley, Anodic oxidation of aluminium and its alloys, Pergamon Press, Oxford, 1st edition, 1982.

[3] Y. Cheng, J. Cao, Z. Peng et al., "Wear-resistant coatings formed on Zircaloy-2 by plasma electrolytic oxidation in sodium aluminate electrolytes," Electrochimica Acta, vol. 116, pp. 453466, 2014.

[4] E. Matykina, R. Arrabal, D. J. Scurr, A. Baron, P. Skeldon, and G. E. Thompson, "Investigation of the mechanism of plasma electrolytic oxidation of aluminium using $18 \mathrm{O}$ tracer," Corrosion Science, vol. 52, no. 3, pp. 1070-1076, 2010.

[5] Y. Xu, Z. Yao, F. Jia, Y. Wang, Z. Jiang, and H. Bu, "Preparation of PEO ceramic coating on Ti alloy and its high temperature oxidation resistance," Current Applied Physics, vol. 10, no. 2, pp. 698-702, 2010.

[6] J. A. Curran and T. W. Clyne, "Thermo-physical properties of plasma electrolytic oxide coatings on aluminium," Surface and Coatings Technology, vol. 199, no. 2-3, pp. 168-176, 2005.

[7] Y. Zhong, L. Shi, M. Li, F. He, and X. He, "Characterization and thermal shock behavior of composite ceramic coating doped with $\mathrm{ZrO} 2$ particles on TC4 by micro-arc oxidation," Applied Surface Science, vol. 311, pp. 158-163, 2014.

[8] H. Wu, X. Zhang, Z. Geng et al., "Preparation, antibacterial effects and corrosion resistant of porous $\mathrm{Cu}-\mathrm{TiO} 2$ coatings," Applied Surface Science, vol. 308, pp. 43-49, 2014.

[9] M. Fazel, H. R. Salimijazi, M. A. Golozar, and M. R. Garsivaz Jazi, "A comparison of corrosion, tribocorrosion and electrochemical impedance properties of pure $\mathrm{Ti}$ and Ti6Al4V alloy treated by micro-arc oxidation process," Applied Surface Science, vol. 324, pp. 751-756, 2015.

[10] S. L. Aktuğ, S. Durdu, I. Kutbay, and M. Usta, "Effect of $\mathrm{Na}_{2} \mathrm{SiO}_{3} \cdot 5 \mathrm{H}_{2} \mathrm{O}$ concentration on microstructure and mechanical properties of plasma electrolytic oxide coatings on AZ31 $\mathrm{Mg}$ alloy produced by twin roll casting," Ceram Int, vol. 42, pp. 1246-1253, 2016.

[11] S.-C. Yeh, D.-S. Tsai, J.-M. Wang, and C.-C. Chou, "Coloration of the aluminum alloy surface with dye emulsions while growing a plasma electrolytic oxide layer," Surface and Coatings Technology, vol. 287, pp. 61-66, 2016.

[12] L. O. Snizhko, A. L. Yerokhin, A. Pilkington et al., "Anodic processes in plasma electrolytic oxidation of aluminium in alkaline solutions," Electrochimica Acta, vol. 49, no. 13, pp. 20852095, 2004.

[13] L. Xia, J. Han, S. Yang, Z. Yang, and W. Li, "Growth process of scanning microarc oxidation coatings on a356 alloy and their corrosion resistance," Chinese Journal of Materials Research, vol. 30, pp. 814-920, 2016 (Chinese).

[14] V. N. Khromov, J. A. Kuznetsov, and A. N. Novikov, Russian Patent, RU2163272, 1999.

[15] A. D. Pogrebnjak and Y. N. Tyurin, "The structure and properties of $\mathrm{Al} 2 \mathrm{O} 3$ and $\mathrm{Al}$ coatings deposited by microarc oxidation 
on graphite substrates," Technical Physics, vol. 49, no. 8, pp. 1064-1067, 2004.

[16] L. Wen, Y. Wang, Y. Jin, B. Liu, Y. Zhou, and D. Sun, "Microarc oxidation of $2024 \mathrm{Al}$ alloy using spraying polar and its influence on microstructure and corrosion behavior," Surface and Coatings Technology, vol. 228, pp. 92-99, 2013.

[17] J. Martin, A. Melhem, I. Shchedrina et al., "Effects of electrical parameters on plasma electrolytic oxidation of aluminium," Surface and Coatings Technology, vol. 221, pp. 70-76, 2013.

[18] I. Prasertsung, R. Mongkolnavin, S. Damrongsakkul, and C. S. Wong, "Surface modification of dehydrothermal crosslinked gelatin film using a $50 \mathrm{~Hz}$ oxygen glow discharge," Surface and Coatings Technology, vol. 205, supplement 1, pp. S133-S138, 2010.

[19] D. D. DiBitonto, P. T. Eubank, M. R. Patel, and M. A. Barrufet, "Theoretical models of the electrical discharge machining process. I. A simple cathode erosion model," Journal of Applied Physics, vol. 66, no. 9, pp. 4095-4103, 1989.

[20] M. R. Patel, M. A. Barrufet, P. T. Eubank, and D. D. DiBitonto, "Theoretical models of the electrical discharge machining process. II. The anode erosion model," Journal of Applied Physics, vol. 66, no. 9, pp. 4104-4111, 1989.

[21] P. T. Eubank, M. R. Patel, M. A. Barrufet, and B. Bozkurt, "Theoretical models of the electrical discharge machining process. III. the variable mass, cylindrical plasma model," Journal of Applied Physics, vol. 73, no. 11, pp. 7900-7909, 1993.

[22] S. Das, M. Klotz, and F. Klocke, "EDM simulation: Finite element-based calculation of deformation, microstructure and residual stresses," Journal of Materials Processing Technology, vol. 142, no. 2, pp. 434-451, 2003.

[23] J. Tao, J. Ni, and A. J. Shih, "Modeling of the Anode Crater Formation in Electrical Discharge Machining," Journal of Manufacturing Science and Engineering, vol. 134, pp. 192-197, 2012.

[24] C. B. Wei, X. B. Tian, S. Q. Yang, X. B. Wang, R. K. Y. Fu, and P. K. Chu, "Anode current effects in plasma electrolytic oxidation," Surface and Coatings Technology, vol. 201, no. 9-11, pp. 50215024, 2007.

[25] T. Wei, F. Yan, and J. Tian, "Characterization and wear- and corrosion-resistance of microarc oxidation ceramic coatings on aluminum alloy," Journal of Alloys and Compounds, vol. 389, no. 1-2, pp. 169-176, 2005.

[26] H.-H. Wu, Z.-S. Jin, B.-Y. Long, F.-R. Yu, and X.-Y. Lu, "Characterization of microarc oxidation process on aluminium alloy," Chinese Physics Letters, vol. 20, no. 10, pp. 1815-1818, 2003.

[27] R. McPherson, "Formation of metastable phases in flame- and plasma-prepared alumina," Journal of Materials Science, vol. 8, no. 6 , pp. 851-858, 1973.

[28] J. Li, H. Cai, X. Xue, and B. Jiang, “The outward-inward growth behavior of microarc oxidation coatings in phosphate and silicate solution," Materials Letters, vol. 64, no. 19, pp. 2102-2104, 2010.

[29] Z. Shi, G. Song, and A. Atrens, "Influence of anodising current on the corrosion resistance of anodised AZ91D magnesium alloy," Corrosion Science, vol. 48, no. 8, pp. 1939-1959, 2006.

[30] L. Zhu, Z. Guo, Y. Zhang, Z. Li, and M. Sui, "A mechanism for the growth of a plasma electrolytic oxide coating on Al," Electrochimica Acta, vol. 208, pp. 296-303, 2016.

[31] J.-H. Wang, M.-H. Du, F.-Z. Han, and J. Yang, "Effects of the ratio of anodic and cathodic currents on the characteristics of micro-arc oxidation ceramic coatings on $\mathrm{Al}$ alloys," Applied Surface Science, vol. 292, pp. 658-664, 2014.
[32] M. Tang, W. Li, H. Liu, and L. Zhu, "Influence of titania sol in the electrolyte on characteristics of the microarc oxidation coating formed on 2A70 aluminum alloy," Surface and Coatings Technology, vol. 205, no. 17-18, pp. 4135-4140, 2011.

[33] R. H. U. Khan, A. Yerokhin, X. Li, H. Dong, and A. Matthews, "Surface characterisation of DC plasma electrolytic oxidation treated 6082 aluminium alloy: Effect of current density and electrolyte concentration," Surface and Coatings Technology, vol. 205, no. 6, pp. 1679-1688, 2010. 

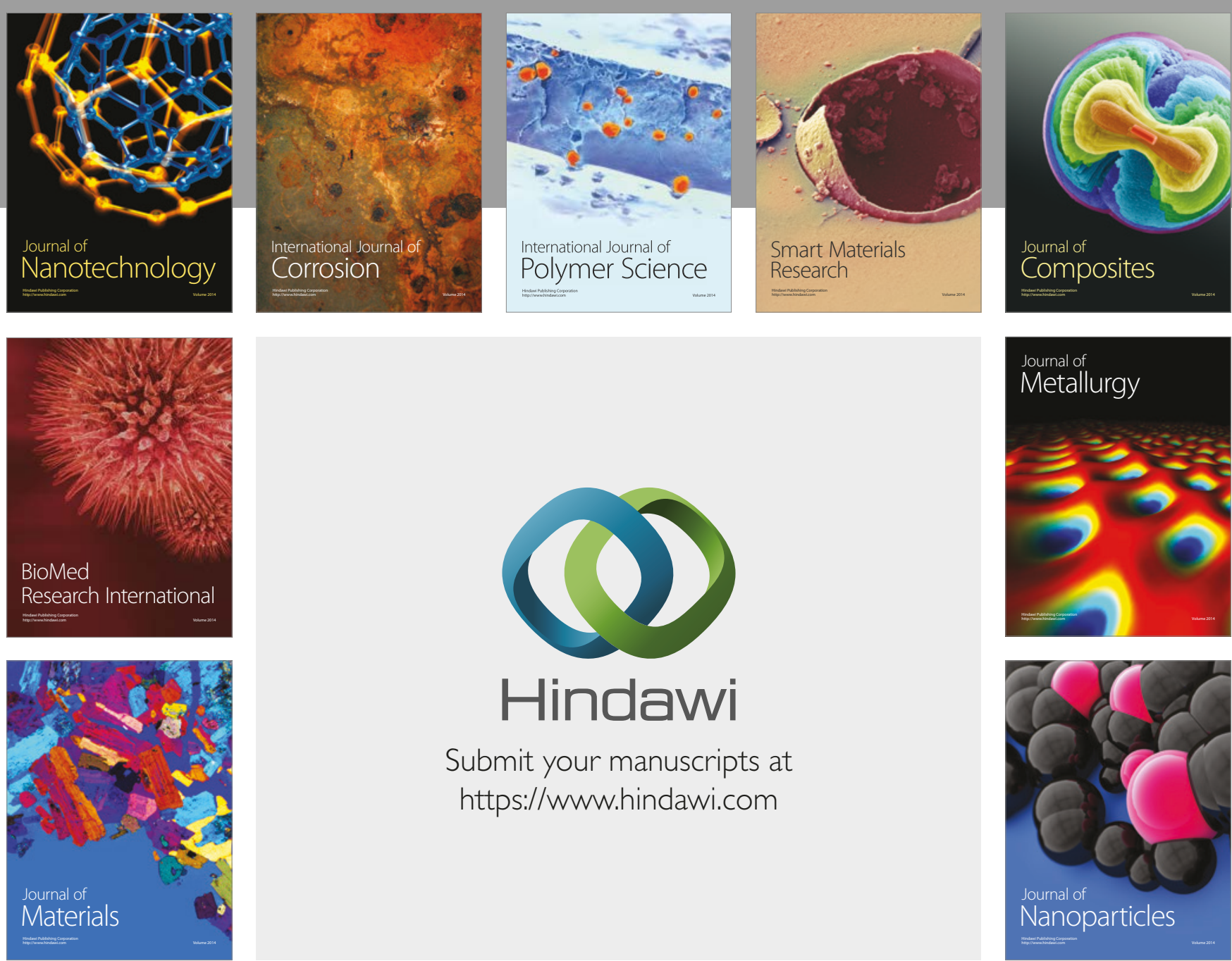

\section{Hindawi}

Submit your manuscripts at

https://www.hindawi.com
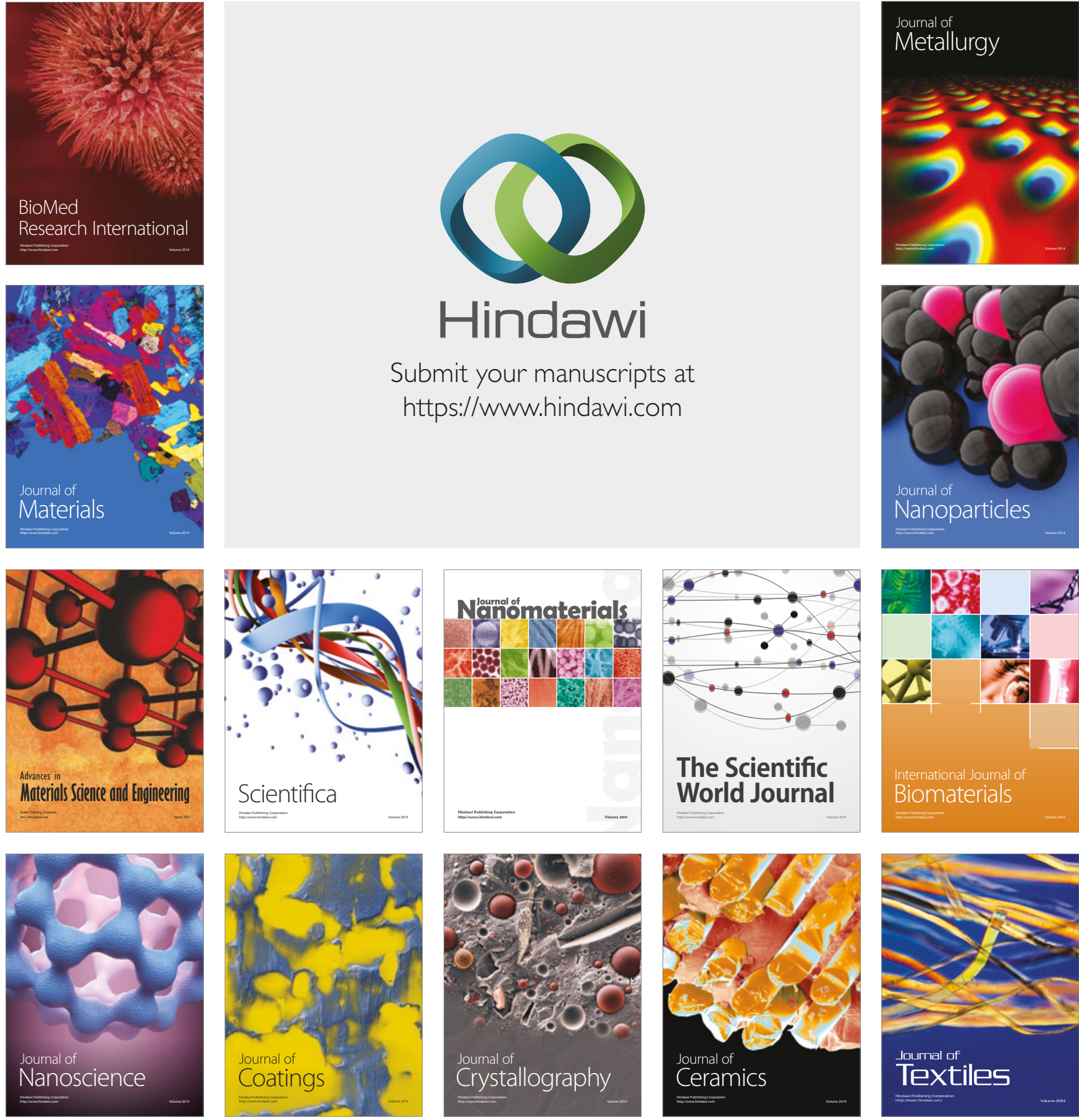

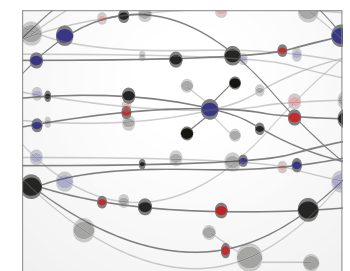

The Scientific World Journal
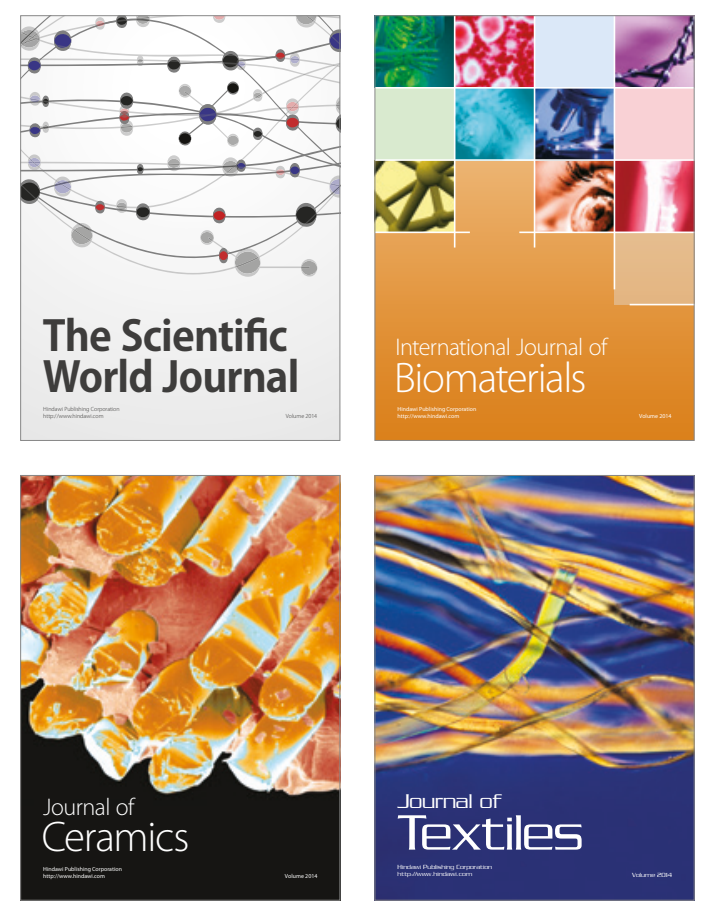